\title{
Human immunodeficiency virus (HIV)-associated duodenal lymphoma
}

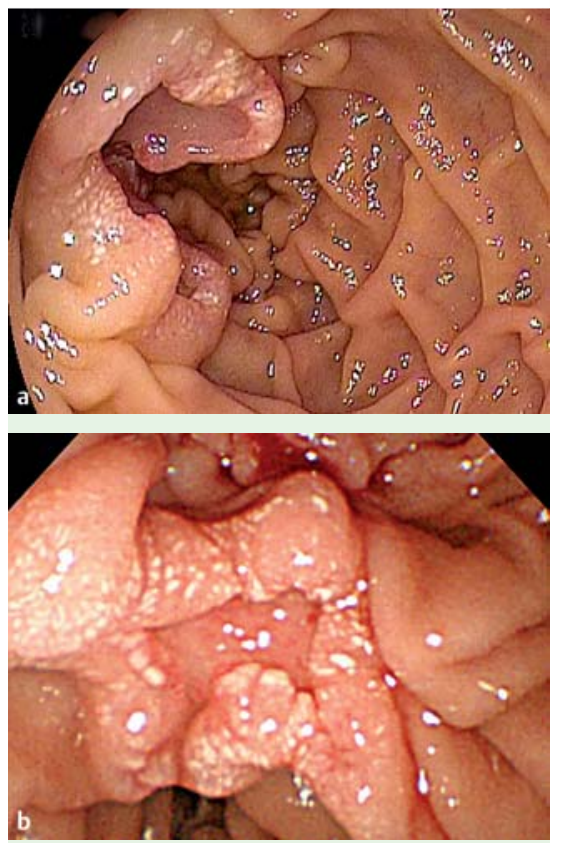

Fig. 1 Endoscopic images of the duodenum showing two ulcerative lesions: a second part of the duodenum; $\mathbf{b}$ inferior duodenal angulus.

A 68-year-old man was referred to our hospital for HIV treatment. He noticed melena on admission. Laboratory data showed anemia, with a hemoglobin level of $8.2 \mathrm{~g} / \mathrm{dL}$. The CD4 lymphocyte count was 101 cells $/ \mu \mathrm{L}$.

Esophagogastroduodenoscopy

(EGD)

showed two ulcerative lesions in the second part of the duodenum and in the inferior duodenal angulus ( Fig. 1). These well-demarcated lesions consisted of an ulcer with a clean base and a regular elevated margin that had an auricle-like shape and many scattered tiny white spots. Histological examination of the biopsy specimens confirmed the diagnosis of diffuse large B-cell lymphoma (DLBCL). Immunochemical staining was positive for L-26 (CD20) ( $\bullet$ Fig. 2).

Antiretroviral therapy (ART) was started for HIV treatment. After ART, the patient received six cycles of $\mathrm{R}-\mathrm{CHOP}$ (rituximab, cyclophosphamide, doxorubicin, vincristine, and prednisolone) chemotherapy. Follow-up EGD showed tumor regression. Non-Hodgkin's lymphoma is a common malignancy in HIV-positive individuals,

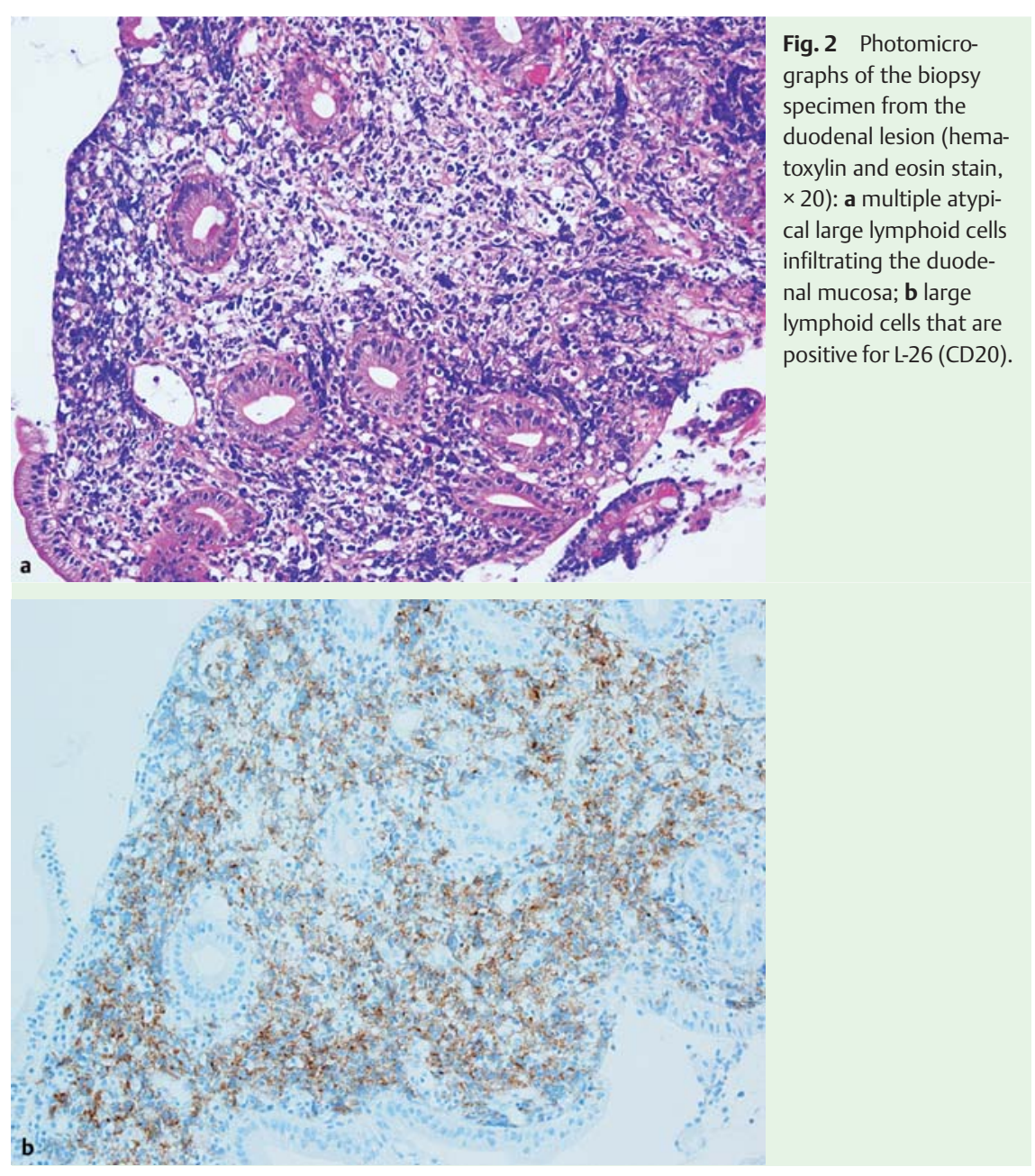

and the gastrointestinal tract is one of the most common extranodal sites. However, HIV-associated duodenal lymphomas are very rare [1-3]. The endoscopic findings in AIDS-associated lymphoma are reported to be similar to those for gastrointestinal lymphomas in immunocompetent patients: polypoid, bulky lesions or well-defined ulcers are found in combination or as single lesions [4]. The most common HIV-associated lymphomas are Burkitt's lymphoma and DLBCL. Although the incidence of follicular lymphoma in the duodenum is relatively higher than in other portions of the gastrointestinal tract [5], follicular lymphoma has not been reported in HIV-infected patients. Our patient was diagnosed with DLBCL and showed an excellent response to the R-CHOP chemotherapy administered after ART.

If unique duodenal ulcerative lesions are found, HIV-associated duodenal lymphoma should be considered as a rare differential diagnosis. Furthermore, accurately diagnosing DLBCLs by endoscopic biopsy is very important in such cases because HIV-associated lymphomas of the gastrointestinal tract have a poor prognosis [4] and require aggressive treatment.

Endoscopy_UCTN_Code_CCL_1AB_2AZ_3AB

Competing interests: None 
S. Nakazuru', T. Yoshio', Y. Ogawa², K. Yuguchi ${ }^{1}$, H. Hasegawa', Y. Sakakibara $^{1}$, Y. Kodama ${ }^{3}$, T. Uehira ${ }^{4}$, E. Mita ${ }^{1}$

1 Department of Gastroenterology and Hepatology, National Hospital Organization, Osaka National Hospital, Osaka, Japan

2 Department of Hematology, Osaka City General Hospital, Osaka, Japan

${ }^{3}$ Department of Pathology, National Hospital Organization, Osaka National Hospital, Osaka, Japan

${ }^{4}$ AIDS Medical Center, National Hospital Organization, Osaka National Hospital, Osaka, Japan

\section{References}

1 Bécheur H, Piketty C, Bloch Fet al. Endoscopic diagnosis of a duodenocolic fistula due to a non-Hodgkin's lymphoma in a patient with AIDS. Endoscopy 1996; 28: 528-529

2 Corti M, Villafañe MF, Souto L et al. Burkitt's lymphoma of the duodenum in a patient with AIDS. Rev Soc Bras Med Trop 2007; 40: $338-340$

3 Andhavarapu S, Tolentino AM, Jha C et al. Diffuse large B-cell lymphoma presenting as multiple lymphomatous polyposis of the gastrointestinal tract. Clin Lymphoma Myeloma 2008; 8: 179-183

4 Heise W, Arastéh K, Mostertz P et al. Malignant gastrointestinal lymphomas in patients with AIDS. Digestion 1997; 58: 218-224

5 Yoshino T, Miyake K, Ichimura $K$ et al. Increased incidence of follicular lymphoma in the duodenum. Am J Surg Pathol 2000; 24: $688-693$
Bibliography

DOI $10.1055 / \mathrm{s}-0030-1256940$

Endoscopy 2011; 43: E384-E385

(c) Georg Thieme Verlag KG Stuttgart · New York . ISSN 0013-726X

\section{Corresponding author}

\section{S. Nakazuru, MD}

Department of Gastroenterology and Hepatology National Hospital Organization

Osaka National Hospital

2-1-14 Houenzaka

Chuo-ku

Osaka City

Osaka 540-0006

Japan

Fax: +81-6-69463569

nakazuru@onh.go.jp 